\title{
Native T1 mapping in patients with idiopathic dilated cardiomyopathy for the assessment of diffuse myocardial fibrosis: validation against histologic endomyocardial biopsy
}

Yoshitaka Goto ${ }^{1 *}$, Masaki Ishida ${ }^{1}$, Shiro Nakamori' ${ }^{2}$, Motonori Nagata', Yasutaka Ichikawa', Kakuya Kitagawa', Kaoru Dohi ${ }^{2}$, Masaaki Ito², Hajime Sakuma ${ }^{1}$

From 18th Annual SCMR Scientific Sessions

Nice, France. 4-7 February 2015

\section{Background}

Late gadolinium enhancement (LGE) MRI provides a significant impact on prognosis in dilated cardiomyopathy (DCM) patients. However, LGE MRI is less suitable for quantifying the degree of fibrosis in diffusely diseased myocardium. T1 mapping technique allows for the quantitative assessment of extracellular volume fraction (ECV), which has been histologically validated against the collagen volume fraction (CVF). Native myocardial T1 also has a potential for the noninvasive detection of myocardial fibrosis. Recent study demonstrated that native myocardial $\mathrm{T} 1$ permits the discrimination between normal and diffusely diseased myocardium accurately in DCM patients. However, in-vivo histological validation of native myocardial T1 in DCM patients is still lacking. The aim of this study was to histologically validate native myocardial $\mathrm{T} 1 \mathrm{for}$ the assessment of diffuse myocardial fibrosis in DCM patients.

\section{Methods}

Twenty DCM patients (18 men, $56.8 \pm 15.7$ years old) underwent CMR including cine, LGE MRI and pre- and post-contrast T1 mapping using a modified Look-Locker inversion recovery sequence at 3T. A significant coronary artery disease was excluded by invasive coronary angiography in all patients. All patients underwent endomyocardial biopsies from right septal ventricle. The diagnosis of DCM was confirmed histologically in all patients. T1 values were quantified within the septal

\footnotetext{
'Radiology, Mie University Hospital, Tsu, Japan

Full list of author information is available at the end of the article myocardium and LV blood pool with a heart rate correction. ECV was calculated from myocardial and blood T1 measured before and after the administration of gadolinium contrast medium and hematocrit measures. CVF was quantified histologically from biopsy specimens using picrosirius red staining.

\section{Results}

The CVF, native T1 and ECV was $16.4 \pm 11.5 \%$ (range, $4 \%$ to $50 \%$ ), $1398.0 \pm 55.1 \mathrm{~ms}$ (range, $1249.9 \mathrm{~ms}$ to $1491.8 \mathrm{~ms}$ ) and $32.6 \pm 7.2 \%$ (range, $22.7 \%$ to $58.7 \%$ ), respectively. LGE was observed in 5 of the 20 patients on LGE MRI. Both CVF and native $\mathrm{T} 1$ were significantly greater in the patients with LGE than those without LGE (CFV, $27.4 \pm 15.0$ vs $12.7 \pm 8.1 \%, \mathrm{p}=0.011$; native $\mathrm{T} 1,1446.8 \pm 29.3$ $\mathrm{ms}$ vs $1381.7 \pm 54.3 \mathrm{~ms}, \mathrm{p}=0.021)$. Although the ECV tended to be grater in the patients with LGE than those without LGE, the difference was not significant $(39.6 \pm 111.4 \%$ vs $30.3 \pm 3.6 \%, p=0.147)$. Both ECV and native T1 were strongly associated with CVF $(y=0.443 x$ $+25.4, \mathrm{r}=0.711, \mathrm{p}<0.001$ and $\mathrm{y}=3.225 \mathrm{x}+1345.3, \mathrm{r}=0.673$, $\mathrm{p}=0.001$, respectively). Inter- and Intra- observer reproducibility for nativeT1 and ECV were 0.903, 0.977, 0.942 and 0.988 , respectively. Figure 1.

\section{Conclusions}

The current results demonstrated that both native T1 and ECV have a good correlation with histological collagen fraction in DCM patients. Native T1 mapping may serve as a noninvasive technique that allows for quantitative assessment of diffuse myocardial fibrosis in DCM 


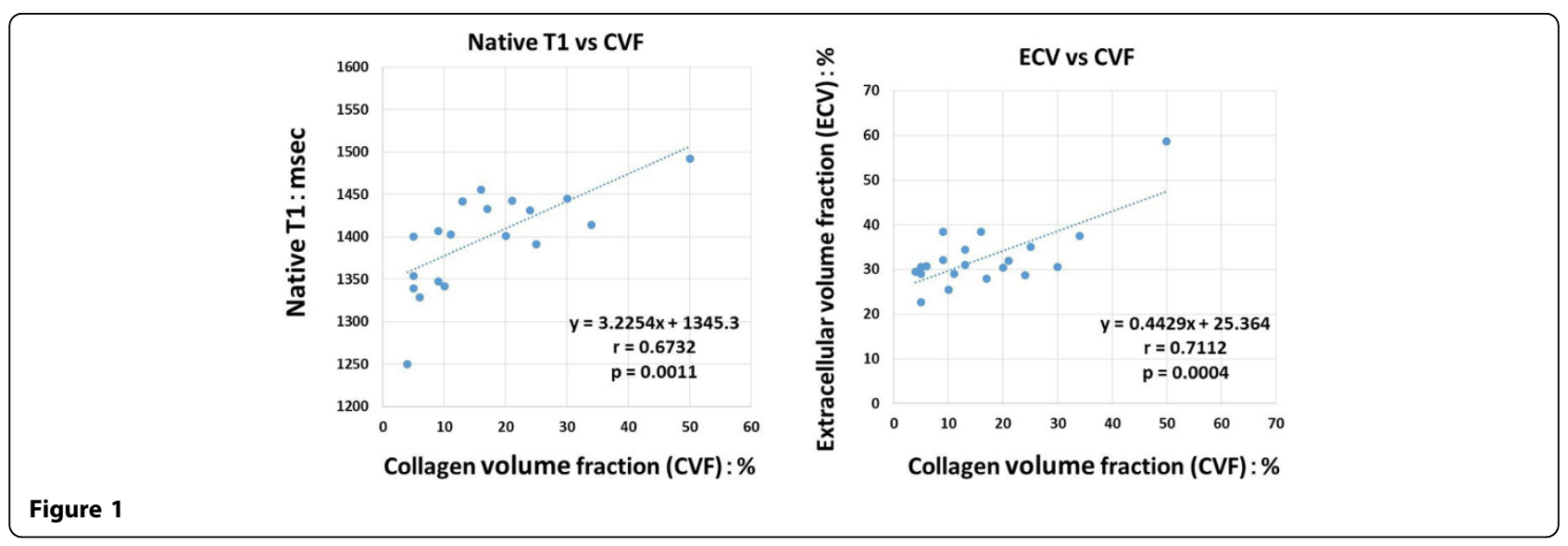

patients without administrating gadolinium contrast medium.

\section{Funding}

N/A.

\section{Authors' details}

${ }^{1}$ Radiology, Mie University Hospital, Tsu, Japan. ${ }^{2}$ Cardiology, Mie University

Hospital, Tsu, Japan.

Published: 3 February 2015

- Convenient online submission

- Thorough peer review

- No space constraints or color figure charges

- Immediate publication on acceptance

- Inclusion in PubMed, CAS, Scopus and Google Scholar

- Research which is freely available for redistribution 